\title{
Pachymeningitis in a Patient With Granulomatosis With Polyangiitis; A Case Report
}

\author{
Gholam Hossein Alishiri $^{10}$, Ehsan Rahmanian ${ }^{2 *}$, Mahsa Ramezanpour ${ }^{2}$ \\ ${ }^{1}$ Chemical Injuries Research Center, Systems Biology and Poisonings Institute, Department of Rheumatology, Faculty \\ of Medicine, University of Medical Sciences, Tehran, Iran \\ ${ }^{2}$ Student Research Committee, Department of Rheumatology, Faculty of Medicine, Baqiyatallah University of Medical \\ Sciences, Tehran, Iran
}

*Corresponding Author: Ehsan Rahmanian, M.D., Student Research Committee, Department of Rheumatology, Faculty of Medicine, Baqiyatallah University of Medical Sciences, Tehran, Iran. Tel: +98-3538241621, Email: drrahmanianehsan@yahoo.com

Received November 6, 2020; Accepted February 8, 2021; Online Published March 7, 2021

\begin{abstract}
Introduction: Granulomatosis with polyangiitis (GPA) is a systematic and necrotizing vasculitis with positive autoimmune antibodies. Some studies have reported the prevalence of eye involvement between $40 \%-50 \%$ of cases. Retro orbital granuloma is a rare complication of GPA which should be treated by surgical involvements, while pachymeningitis can be diagnosed by MRI and treated by medical management. In this study, we tried to present a case of GPA with optic neuritis and typical central nervous system (CNS) involvement, while there were no definite features of sinusitis or kidney injuries.

Case Presentation: A 15-year-old girl was admitted because of blurred vision in her left eye. She was a known case of GPA three years ago with initial features, including left facial nerve paresis due to pan-sinusitis and pulmonary cavity. Neurologic evaluations, including sensory and motor features, were normal, too. Ophthalmologic examinations showed that visual acuity of the right eye was good, while the visual acuity in the left eye decreased to the point of finger counting at a distance of $20 \mathrm{~cm}$. The left eye Marcus gunn test was positive (3+); anterior and posterior eye segments were normal. The patient was evaluated by brain MRI with gadolinium and a pathologic enhancement in the left cavernous was seen which had a pressure effect on the optic nerve. She was treated by intravenous methylprednisolone followed by rituximab.

Conclusion: Reporting orbital mass in a patient who had GPA can be supposed as granuloma which needs a biopsy to confirm a diagnosis. In our case, the imaging manifestation was heterodox for granuloma, while neurosurgical consultation recommended drug treatment for pachymeningitis.

Keywords: GPA, Pachymeningitis, Granuloma, Orbit
\end{abstract}

\section{Introduction}

Granulomatosis with polyangiitis (GPA) is a systematic and necrotizing vasculitis with positive autoimmune antibodies whose most common presentation is upper respiratory involvement with hematuria. ${ }^{1}$ Some studies have reported the prevalence of eye involvement between $40 \%-50 \%$ of cases. Some complications are rare in this disorder, including central nervous system (CNS) involvement especially optic neuritis, cranial nerve paralysis, pachymeningitis, or pituitary disorders while in some cases the presentation of the optic nerve disease was the first feature..$^{2-5}$

Management protocols, including corticosteroid and cyclophosphamide or rituximab therapy, can control the progress of the disease. Medical treatments are the mainstay in ocular or CNS involvement, too. ${ }^{6}$

Retro orbital granuloma is a rare complication of GPA that should be treated by surgical involvements, while pachymeningitis can be diagnosed by MRI and treated by medical management. ${ }^{7,8}$

Similar previous studies showed that optic nerve involvement due to CNS disease can be the complication of this disease and may be treated by controlling GPA. In this study, we tried to present a case of GPA with optic neuritis and typical CNS involvement, while there were no definite features of sinusitis or kidney injuries.

\section{Case Presentation}

A 15-year-old girl was admitted to Baghiatollah Hospital, Tehran, Iran because of blurred vision in the left eye approximately ten days ago. In her past medical history, the patient did not mention any history of head or craniofacial trauma, and no diplopia, paresthesia, or paresis was detected.

She was a known case of GPA three years ago with initial features, including left facial nerve paresis due to pan-

Copyright (C 2021 The Author(s). This is an open-access article distributed under the terms of the Creative Commons Attribution License (http:// creativecommons.org/licenses/by/4.0), which permits unrestricted use, distribution, and reproduction in any medium, provided the original work is properly cited. 
sinusitis and pulmonary cavity. The disease was controlled by intravenous methylprednisolone and after remission, she was followed by taking $10 \mathrm{mg}$ of oral prednisolone and $100 \mathrm{mg}$ of azathioprine daily.

The first evaluations in the emergency department did not show any fever and the other systematic exams were approximately normal. Neurologic evaluations, including sensory and motor features, were normal, too. The muscle force of upper and lower extremities was good. Ophthalmologic examinations showed that visual acuity of the right eye was good, while the visual acuity in the left eye decreased to the point of finger counting at a distance of $20 \mathrm{~cm}$. The left eye Marcus gunn test was positive $(3+)$, anterior and posterior eye segments were normal. The ophthalmologist recommended $250 \mathrm{mg}$ intravenous methylprednisolone four times a day for three days according to retrobulbar optic neuritis.

The patient was evaluated by brain Magnetic resonance imaging (MRI) with gadolinium and a pathologic enhancement in the left cavernous was seen which had a pressure effect on the optic nerve (Figure 1). Initial reports of imaging recommended retro-orbital mass which can be diagnosed as an orbital pseudo tumor, including granuloma or pachymeningitis. By neurosurgery consults, these findings proposed pachymeningitis in the left cavernous sinuous, and treatment started without biopsy. Other Para clinic findings showed normal complete blood count parameters and renal function tests. Erythrocyte sedimentation rate (ESR) was raised to 27 and C-reactive

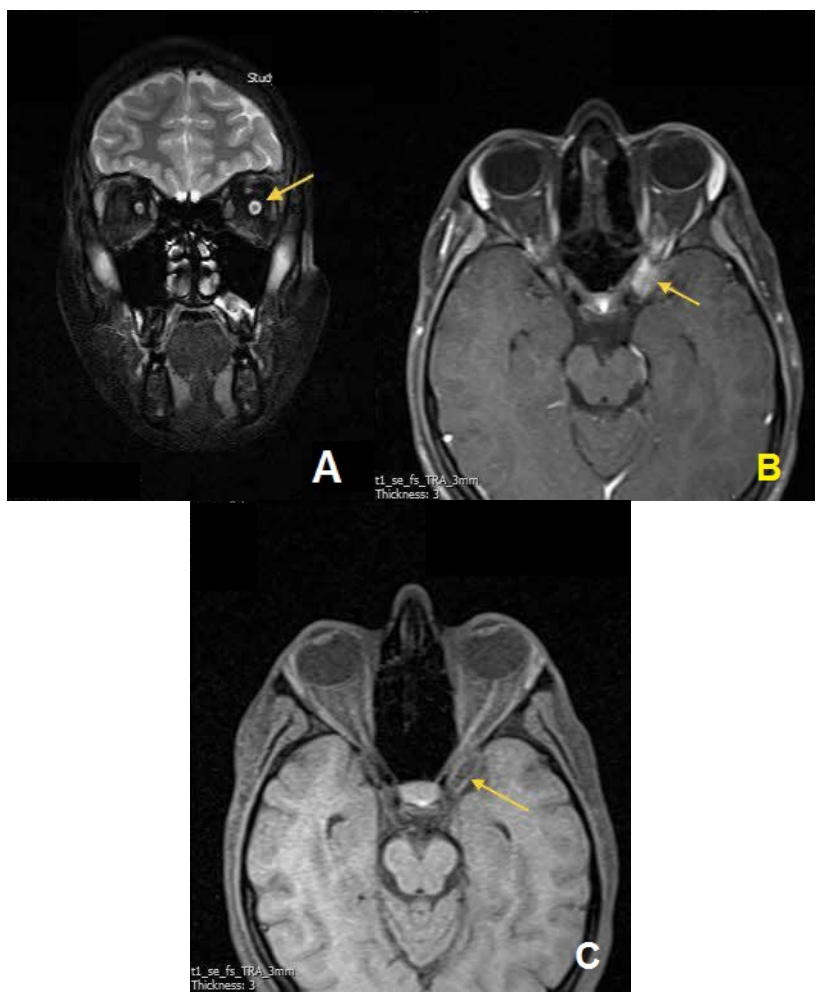

Figure 1. Left Optic Nerve Inflammation and Edema (Retrobulbar Neuritis). (A) Inversion recovery sequence of MRI; (B) Axial STIR inversion recovery sequence of $\mathrm{MRI}$; $(\mathrm{C})$ Optic nerve enhancement after gadolinium injection. protein (CRP) was 5.7. PPD and IGRA (interferon gamma release assay) test was negative. Urine analysis showed some leukocyturia (4-5 WBC) without hematuria.

She was treated with intravenous methylprednisolone for three days which was followed by rituximab. Because of her young age and the risk of infertility, cyclophosphamide was not prescribed to her. In the first days of treatment, the blurred vision was better, and in regular ophthalmologic examinations in admission days and six months after discharge was normal, too. In follow-up MRI, the CNS lesions were revealed.

\section{Discussion}

GPA may have some rare manifestations, one of which is optic neuritis due to patchy CNS involvements. ${ }^{4-6}$ In this case report, the patient was under maintenance treatment before initiation of ocular treatment and may be supposed as the flare-up of the disease. Due to these rare manifestations, the case was presented for further training. Another reason why this case was presented was the use of medical treatment rather than surgical treatment or radiotherapies.

One of the important aspects which convinced us to report this case was its imaging manifestation. Reporting orbital mass in a patient who had a past medical history of GPA can be supposed as granuloma which needs a biopsy to confirm the diagnosis. Tuberculosis is one of the most important granulomatosis mass in such patients.in our case, the imaging manifestation was heterodox for granuloma, while neurosurgery consultation recommended drug treatment for pachymeningitis.

Bitik et al reported a case series that had retroorbital granuloma due to GPA. They recommended surgical interventions to decrease mortality and serious complications. ${ }^{7}$ Smoleńska etal introduced pachymeningitis as a rare complication of GPA. They recommended proper diagnosis by MRI and accurate management by medical treatment to control the progression of complications. ${ }^{8}$

\section{Conclusion}

The current study showed optic neuritis and a mass in the optic nerve. We treated the vasculitis with a high dose of glucocorticoid and Rituximab that the patient showed a good response to them. Thus, we treated patchy meningitis or brain pseudotumor with proper agents of vasculitis treatment and not surgery.

\section{Authors' Contributions}

All Authors contributed equally to this study.

\section{Conflict of Interest Disclosures}

Gholam Hossein Alishiri serves as a editor in chief of "Hospital Practices and Research". Other authors declare that they have no conflicts of interest.

\section{Ethical Approval}

The patient gave permission for the presentation of his case reports. 


\section{Acknowledgments}

The authors would like to thank the "Clinical Research Development Unit" of Baqiyatallah Hospital for their kind cooperation.

\section{References}

1. de Groot K, Schmidt DK, Arlt AC, Gross WL, ReinholdKeller E. Standardized neurologic evaluations of 128 patients with Wegener granulomatosis. Arch Neurol. 2001;58(8):1215-1221. doi:10.1001/archneur.58.8.1215.

2. Moubayed SP, Black DO. Optic neuritis as an initial presentation of Wegener's granulomatosis. Can J Ophthalmol. 2009;44(6):e59. doi:10.3129/i09-145.

3. Lutalo PM, D'Cruz DP. Diagnosis and classification of granulomatosis with polyangiitis (aka Wegener's granulomatosis). J Autoimmun. 2014;48-49:94-8. doi: 10.1016/j.jaut.2014.01.028.

4. Haynes BF, Fishman ML, Fauci AS, Wolff SM. The ocular manifestations of Wegener's granulomatosis. Fifteen years experience and review of the literature. Am J Med. 1977;63(1):131-141. doi:10.1016/0002-9343(77)90125-5.

5. Seror R, Mahr A, Ramanoelina J, Pagnoux C, Cohen P, Guillevin L. Central nervous system involvement in Wegener granulomatosis. Medicine (Baltimore). 2006;85(1):53-65. doi:10.1097/01.md.0000200166.90373.41.

6. Babar L, Bakalov V, Abel S, et al. Retrospective review of total neoadjuvant therapy. World J Gastrointest Oncol. 2019;11(10):857-865. doi:10.4251/wjgo.v11.i10.857.

7. Bitik B, Kılıç L, Küçükşahin O, et al. Retro-orbital granuloma associated with granulomatosis with polyangiitis: a series of nine cases. Rheumatol Int. 2015;35(6):1083-1092. doi:10.1007/s00296-014-3179-8.

8. Smoleńska Ż, Masiak A, Zdrojewski Z. Hypertrophic pachymeningitis as an important neurological complication of granulomatosis with polyangiitis. Reumatologia. 2018;56(6):399-405. doi:10.5114/reum.2018.80719. 